\title{
MECHANIZED INFORMATION RETRIEVAL
}

An international program for mechanized information retrieval on pulp and paper problems entered an active development phase on January 1, 1962.

Dr. Lincoln R. Thiesmeyer, President of the Pulp and Paper Research Institute of Canada, and Dr. John G. Strange, President of The Institute of Paper Chemistry, are maintaining close contact during all phases of development of the information and literature retrieval systems so that what is eventually made available to the industries in each country will be fully compatible.

The problem with the traditional library index is that it does not provide sufficiently detailed classification of information and is too inflexible to meet the needs of constantly changing technologies. Of the many approaches to this problem, the one currently receiving the most attention is the method of keywords to characterize the contents of any document. This group of words can be large enough to characterize the document in any required detail, and changes in technology can be reflected by the addition of necessary words to the keyword dictionary. A query for information is also stated in keywords, which characterize the subject under investigation. The information search is made by selecting documents which have keywords matching those of the query.

The central core of information about which this system is to be built is the Abstract Bulletin of The Institute of Paper Chemistry, which annually cites, abstracts and indexes some 10,000 technical papers, patents and review articles of interest to the pulp and paper industry. Over 800 different publications originating in 31 countries are searched by IPC staff members in the collection of this information. In Canada, additional journals in the pulp and paper field, and particularly in the forestry field, are similarly searched by PPRI staff. For internal member company use, each institution will have individual, confidential supplements to the main file.

During the first six months of 1962, PPRI and IPC will be conducting separate, but co-ordinated, intensive experiments with various information system configurations.

It is planned to use a document file of some 5000 items for this experimentation in order to gain statistically significant data on both indexing (input) and searching (output) problems. Indexing and coding of this file will be accomplished by using the AIChE Chemical Engineering Thesaurus, the index terms of the IPC Abstract Bulletin and a special "keyboard dictionary" of pulp and paper terms, the first draft of which has been prepared by PPRI staff.

It is expected that this experiment will result in a suggested system for pulp and paper industry use, which, in general form, may be employed by any individual or organization at whatever level of complication his needs and facilities indicate. For small, individual collections, a system based on manual use of index cards will suffice. Some large organizations may wish to duplicate the mechanized systems at IPC and PPRI which will center around IBM 1620 data processing systems.

Work at the Pulp and Paper Research Institute of Canada is being conducted by Kenneth E. Vroom, Chairman, Technical Services Department, John E. Tasman, and G. J. Clement Potter. 